\title{
The Impact of Prior Knowledge about Visual Feedback on Motor Performance and Learning
}

\author{
James J. Burkitt ${ }^{1}$, Lawrence E. M. Grierson ${ }^{2}$, Victoria Staite ${ }^{1}$, \\ Digby Elliott ${ }^{1,3}$, James Lyons ${ }^{1}$ \\ ${ }^{1}$ Department of Kinesiology, McMaster University, Hamilton, Canada \\ ${ }^{2}$ Department of Family Medicine, McMaster University, Hamilton, Canada \\ ${ }^{3}$ School of Sport and Exercise Sciences, Liverpool John Moores University, Liverpool, UK \\ Email: burkitjj@mcmaster.ca
}

Received September $21^{\text {st }}$, 2012; revised October $25^{\text {th }}, 2012$; accepted November $8^{\text {th }}, 2012$

\begin{abstract}
Performers adopt strategies to use visual information if they know that it will be available whereas uncertainty about its availability leads performers to prepare for the worst-case scenario. Since the impact of prior knowledge has generally been examined as performance-based changes across a series of trials, this study investigates the impact of prior knowledge on learning. Participants practiced target-directed aiming movements either with cues about the random availability of vision, no cues regarding vision or in blocks of stable visual information availability. Participants who received prior knowledge were more efficient in preparing their acquisition movements when vision was available. In retention and transfer, all participants were able to take advantage of the visual information available in order to optimize performance outcome. Thus, adult performers appear able to change their strategic behavior quickly to accommodate new sensory and prior knowledge conditions.
\end{abstract}

Keywords: Motor Learning; Feedback; Speed-Accuracy; Vision; Aiming

\section{Introduction}

Over 40 years ago, Keele and Posner (1968) published an influential paper in which they estimated it took a minimum of 190 - 260 ms to process and use visual feedback for the regulation of discrete goal-directed aiming. Although this approximation of visual processing time stood up well for a number of years, experiments conducted in the early and mid 1980's resulted in estimates ranging from 140 ms (Elliott \& Allard, 1985) to less than 100 ms (Bard, Hay, \& Fleury, 1985; see Elliott, Helsen, \& Chua, 2001 for review; Zelaznik, Hawkins, \& Kisselburgh, 1983). An important procedural difference between these studies was that Keele and Posner (1968) used a random schedule to manipulate the availability of vision, while Zelaznik et al. (1983) and Elliott and Allard (1985) blocked their visual manipulation. It would appear that, for very rapid visual feedback utilization to occur, it is important the performer knows in advance that visual feedback will be available for online regulation (Elliott \& Allard, 1985; Keele \& Posner, 1968; Zelaznik et al., 1983). When there is uncertainty about the availability of feedback (e.g., a random vision-no vision schedule), the performer prepares for the worst-case, or no vision, scenario (Elliott, Hansen, Mendoza, \& Tremblay, 2004; Hansen, Glazebrook, Anson, Weeks, \& Elliott, 2006; Khan, Elliott, Coull, Chua, \& Lyons, 2002).

This interpretation of outcome findings is consistent with the spatial-temporal characteristics of aiming movements performed with and without vision. These characteristics reflect the strategic approach to movement planning and current control that people take to minimize error and maximize movement speed. Specifically, when the performer knows that vision will not be available for online regulation, he/she generally takes more time to initiate the aiming movement (Khan et al., 2002). Presumably, this extra time reflects more precise movement planning. The velocity profiles under no vision conditions are usually more symmetric than when vision is available. When the performer knows in advance that visual feedback will be available for online regulation, the aiming limb reaches a higher peak velocity earlier in the movement than under no vision conditions. Hansen et al. (2006) suggested that this strategy reflects the performer's attempt to get the limb to the target area quickly in order to take full advantage of visual feedback when the limb is in the target area (see also Elliott, Chua, Pollock, \& Lyons, 1995). This aiming strategy results in greater trial-to-trial spatial variability early in the movement, but also a large decrease in variability between peak deceleration and movement termination, as the limb benefits from late visual information about its trajectory relative to the position of the target (Khan et al., 2002).

When there is uncertainty about the availability of vision for online regulation, the spatial-temporal characteristics of both vision and no vision trials look much like no vision aiming attempts performed under blocked conditions (Hansen et al., 2006). This finding indicates that many of the differences between the characteristics of vision and no vision movements are strategic in nature. Specifically, they reflect the performer's attempt to make full use of the visual and other information they know will be available.

In this study, our objective was to extend our performancebased findings on prior knowledge about feedback to motor learning. Our primary prediction was that when the performer is denied prior knowledge about the availability of vision for online regulation, he/she plans for the worst-case situation. Thus if there is a 50 - 50 chance that vision will be eliminated upon movement initiation, the performer should prepare for the no 
vision circumstance. This strategic approach means that, for a single aiming movement, the performer takes more time to plan the movement and the movement exhibits some of the characteristics of no vision movements, even when vision ends up being available. Over a number of trials in which the performer attempts to improve aiming speed and accuracy, the development of any representation of expected sensory consequences would not include expectancies about visual feedback (Kawato \& Wolpert, 1998; Wolpert \& Flanagan, 2001). It then follows that the proficiency of visual regulation will not develop to the same degree for people trained to rapidly aim to a single target under conditions of feedback uncertainty.

Our research strategy involved assigning participants to one of three groups in which they practiced a series of rapid manual aiming movements to a single target location under different prior knowledge conditions. Two groups received a random vision-no vision feedback schedule. Participants in what we call the Prior Knowledge Group were cued before each trial as to whether or not vision would be available on the upcoming movement. Each participant in the No Prior Knowledge Group was yoked to a single participant in the Prior Knowledge Group. Thus they received exactly the same vision-no vision schedule but were unaware about whether or not to expect visual feedback until the actual movement began. We expected participants in the Prior Knowledge Group to develop separate strategic behaviors for the vision and no vision circumstance that would be reflected in movement kinematics both during practice performance and in a retention and transfer test following task acquisition. Participants in the No Prior Knowledge Group were expected to adopt a strategy consistent with the no vision situation (i.e., worst case situation). Thus any improvement associated with the presence of visual information (i.e., in retention) was expected to be limited. We also included a third group of participants who practiced under blocked feedback conditions. Blocked Feedback participants not only had prior knowledge about what feedback to expect, but over the series of trials they were also able to take advantage of the specific sensory consequences associated with trial $\mathrm{N}$ on trial $\mathrm{N}+1$. Because both sensory feedback and movement outcome on trial $\mathrm{N}$ has a robust effect on trial $\mathrm{N}+1$ performance (Cheng, Luis, \& Tremblay, 2008), we expected participants in this group to benefit more from the prior knowledge about feedback than participants in either of the two random schedule groups.

\section{Methods}

\section{Participants}

Sixty individuals (20 males, 40 females) ranging in age from 17 - 47 years (mean age $=22.6$, sd $=4.81$ ) volunteered to participate in this study. These participants were randomly assigned to one of the 3 practice groups with the constraint that the groups had similar male-female representation. All participants were self-reported right-handers and completed the protocol with their right hand. All participants had normal or corrected to normal vision. Participants were naive to the purpose of the study and all provided written, informed consent prior to participation in accordance with the ethical guidelines of the McMaster University Research Ethics Board.

\section{Apparatus}

Participants were seated at a table upon which was fixed a liquid crystal display (LCD) monitor (Samsung Syncmaster, 910T). The monitor was placed flat on the table so that the screen faced upwards and was positioned in the midline of the participant's body. It was used to display the target location, the prior knowledge information and the movement time feedback, all of which were generated by custom E-Prime software (Psychology Software Tools Inc., Sharpsburg, PA, United States). A piece of clear Plexiglas was fitted over the screen and served as the aiming surface. The reaching movements were performed with a rectangular handheld switch $2.7 \mathrm{~cm}$ in length, $1.0 \mathrm{~cm}$ in width and $1.5 \mathrm{~cm}$ in height. Protruding $1.7 \mathrm{~cm}$ from the front of the switch was a small arm. The arm was connected to the bottom of the switch that closed the switch when in the depressed position and opened the switch when released.

Participants wore liquid crystal goggles (Translucent Technologies; Milgram, 1987) that either provided or occluded vision on a particular trial. These goggles change state in approximately $5 \mathrm{~ms}$. The liquid crystal goggles and the switch were connected to a parallel port that was connected to a computer (ACPI Multiprocessor PC) equipped with E-Prime. Thus, for a no vision trial, vision of the target and the surrounding environment was available as long as the participant kept the arm depressed on the home position. Once the participant moved off the home position and released the switch, vision was occluded. When the switch came in contact with the aiming surface, thereby depressing the arm, vision returned. The time between a change in switch position and an actual change in the state of the goggles was approximately $12 \mathrm{~ms}$. Therefore, vision was occluded only while the limb was in motion. Participants performed the aiming movements with the goal of bringing the arm of the switch in contact with the target. The participants gripped the sides of the switch between their middle finger and thumb, while resting their index finger on top. From this grip, the arm of the switch extended outwards from the hand.

Affixed on the arm of the switch was an infrared light emitting diode (IRED), the displacement of which was captured by an Optotrak 3020 (Northern Digital, Waterloo, ON, Canada). The camera recorded the spatial location of the IRED for $2 \mathrm{~s}$ at $500 \mathrm{~Hz}$. These data were filtered with an $8 \mathrm{~Hz}$ dual-pass Butterworth filter and then run through custom software which differentiated and double differentiated displacement in the primary direction of the movement to provide instantaneous velocity and acceleration.

\section{Procedure}

The protocol consisted of 140 reaching trials, the first 100 of which were acquisition trials and the last 40 of which were retention and transfer trials. In the acquisition trials, participants were asked to make rapid aiming movements from a home position directly in front of them to a circular target with a diameter of $2.0 \mathrm{~cm}$. The target was located $26.67 \mathrm{~cm}$ (10.5 in) away from the home position and both the target and home position were at the participant's midline. Participants were asked to make their aiming movements as quickly and as accurately as possible. All subsequent information relevant to the experiment was displayed to the participant on the monitor by using E-Prime software. The experimenter initiated a trial immediately after the participant depressed the tip of the arm of the switch on the home position. Following a "ready" screen, and dependent upon the condition to which they were randomly 
assigned, participants either received a blank screen or a screen that provided information regarding the type of visual feedback that was going to be available on the proceeding trial. The phrases "vision" and "no vision" were used to provide the latter information. This screen remained on for $500 \mathrm{~ms}$. Following a variable fore-period of $500 \mathrm{~ms}$ to $700 \mathrm{~ms}$, an auditory tone signaled the participant to initiate his/her movement. Movements ended when the arm of the switch came in contact with the aiming surface. Participants were asked to hold the switch at the end position of the movement for a moment after movement termination. During the acquisition phase of the experiment, movement time feedback was provided on the screen prior to the repositioning of the limb at the start position. Participants were instructed to use this feedback to improve their movement time performance. This sequence was repeated for every trial.

During the acquisition trials, participants performed half of the trials with vision (V) and half of the trials without vision $(\mathrm{NV})$. In the $\mathrm{V}$ trials, vision remained throughout the entire duration of the movement, whereas in the NV trials vision was occluded while the limb was in motion. It should be noted that for no vision trials, participants not only lost visual feedback from the moving limb, but also visual information about the position of the target. This situation of course made participants more memory dependent (Elliott \& Madalena, 1987; Henriques, Klier, Smith, Lowry, \& Crawford, 1998).

Participants were randomly assigned to one of three groups that varied with respect to the order in which the $\mathrm{V}$ and $\mathrm{NV}$ trials were performed, and the participant's prior knowledge regarding the type of information ( $\mathrm{V}$ or $\mathrm{NV}$ ) that would be available on the upcoming trial. Specifically, participants in the prior knowledge $(\mathrm{PK})$ group received $\mathrm{V}$ and $\mathrm{NV}$ trials in a randomized manner and received prior knowledge regarding the type of visual information they would be provided on the upcoming trial. Participants in the no prior knowledge (NK) group were yoked to a participant in the PK condition. Thus they also received a random presentation of $\mathrm{V}$ and $\mathrm{NV}$ trials, but without prior knowledge of the visual manipulation on a particular trial. Participants in the blocked prior knowledge (B) group received alternating blocks of $25 \mathrm{~V}$ and $25 \mathrm{NV}$ trials (i.e., 2 blocks of each vision condition). They were informed before the start of a new block about what type of visual information they would receive on the following 25 trials and were also informed prior to the start of each trial as to the type of visual information they would receive on the upcoming trial. The starting order of the $\mathrm{V}$ and NV blocks was counterbalanced across participant. Regardless of the experimental condition, all participants received movement time feedback in the form of knowledge of results (KR) after every trial. This information was provided numerically on the screen following the removal of the target image. Prior to the beginning of the acquisition trials, all participants were instructed to use this movement time information to attempt to improve their movement time performance. Because under no vision conditions the goggles opened with switch contact on or near the target, participants always had terminal feedback about their movement accuracy.

Following the acquisition trials, participants were provided with a five-minute break prior to starting the retention trials. The retention trials followed the same sequence of events as the acquisition trials; participants received a "ready" screen, a screen indicating the type of visual information available on the upcoming trial and then an auditory tone. All participants received prior knowledge regarding the visual conditions of the upcoming trial. The first 20 trials consisted of retention trials (RET), whereby movements were made to the same sized target located in the same position as in the acquisition phase. In the next 20 trials, the small target transfer condition (TRAN), movements were made to a smaller target that was $0.8 \mathrm{~cm}$ in diameter, the center of which was located $26.67 \mathrm{~cm}$ (10.5 in) away from and in line with the home position. No explicit movement time constraints were imposed in either of these two conditions. The post-acquisition trials were the same for all participants such that the ordering of conditions was always RET then TRAN. The V and NV trials were blocked for RET and TRAN. Once again, starting order was counterbalanced across participants. Although participants still received terminal feedback about the position of their limb relative to the target, movement time feedback was not provided.

\section{Data Analysis}

Custom software was used to identify specific kinematic events in each velocity and acceleration profile. The Optotrak frames in which the limb velocity rose above or fell below 30 $\mathrm{mm} / \mathrm{s}$, and remained as such for $70 \mathrm{~ms}$ identified the start and the end of the movement. Reaction time (RT) was the time between the onset of the auditory signal and the beginning of the movement, while movement time (MT) was identified as the time between the beginning and end of the movement. The software identified peak acceleration (PA), peak velocity (PV) and peak deceleration (PD) as well as the time taken to achieve each of these events. It also recorded the spatial location of the limb at each of these 3 kinematic markers, and the end of the movement. These kinematic markers allowed us to examine spatial and temporal central tendency and variability at various points in the movement. The spatial position of the limb at the end of the movement was used to calculate variable error (VE) in the primary direction of the movement. VE is the standard deviation around the mean error. It provides information about within-participant trial-to-trial spatial consistency and is a measure of effective target width (Schmidt, Zelaznik, Hawkins, Frank, \& Quinn, 1979). VE and the percentage of target misses were our two measures of aiming accuracy/consistency.

Trials where the marker was not visible at any point during the movement were omitted from the analysis. Trials in which either the RT or MT for that participant was more than 2.5 standard deviation units from the mean value were also eliminated. Alpha was set at .05 for all inferential analyses.

\section{Results}

\section{Acquisition-Outcome Measures}

In order to determine how participants performed during practice, we partitioned the vision and no vision trials for each of the 3 groups into 5 Blocks of 10 trials each. For the majority of our dependent variables, we performed a 3 Group (PK, NK, B) by 2 Vision Condition (V, NV) by 5 Blocks mixed analysis of variance. Tukey's HSD $(p<.05)$ post hoc procedure was used to decompose any significant effects involving more than two means.

The RT analysis yielded a main effect for Block, $F(4,220)=$ 7.06, $p<.001$, a main effect for Vision Condition, $\mathrm{F}(1,55)=$ $18.09, p<.001$, and a Group by Vision Condition interaction, $\mathrm{F}(2,55)=3.87, p<.05$. As expected, participants became quicker at initiating their movements over practice. The majority 
of this improvement took place in the first block of trials (B1 = $281 \mathrm{~ms}, \mathrm{~B} 2=259 \mathrm{~ms}, \mathrm{~B} 3=255 \mathrm{~ms}, \mathrm{~B} 4=257 \mathrm{~ms}, \mathrm{~B} 5=253$ $\mathrm{ms}$ ). Overall participants took less time to initiate their movements when vision was available (251 $\mathrm{ms})$ than when it was absent (272 ms). As is apparent in Figure 1 however, the difference between $\mathrm{V}$ and NV was only significant for participants in the PK and $\mathrm{B}$ groups. This finding makes sense because only under these conditions do participants know that they will have visual feedback for the online regulation of their aiming movements. Participants in the NK group exhibited similar RTs under vision and no vision conditions. It is interesting to note however that, unlike previous performance studies on prior knowledge, both the V and NV RTs were quite short in the NK group. This overall performance advantage could reflect the fact that participants in the NK group were preparing for the worst-case situation (i.e., no vision) on all 100-acquisition trials. With this fixed/constant preparation strategy, there was no trial-to-trial or block-to-block change in the processing demands.

The MT analysis revealed only a main effect for Block, $\mathrm{F}(4,220)=8.15, p<.001$. Once again participants improved their performance with practice $(\mathrm{B} 1=488 \mathrm{~ms}, \mathrm{~B} 2=473 \mathrm{~ms}$, B3 $=468 \mathrm{~ms}$, B4 $=463 \mathrm{~ms}$, B5 = $460 \mathrm{~ms}$ ). Post hoc analysis revealed only that Block 1 was different from Blocks 3, 4 and 5 $(p<.05)$. Thus the majority of the improvement occurred early in acquisition (see Figure 2).

Because under no vision conditions movement time does not always predict error (Elliott et al., 2001), we also examined the percentage of trials in which participants missed the target. Target misses were considered trials where the end position of the stylus measured greater than $10 \mathrm{~mm}$ from the center of the target in either the primary direction of movement or the direction perpendicular to the primary direction of movement. This analysis yielded only a main effect for Vision Condition, $\mathrm{F}(1,55)$ $=44.43, p<.001$. Participants missed the target on $27 \%$ of their aiming attempts when vision was eliminated upon movement initiation and on $15 \%$ of the trials under full vision. This reasonably high percentage of error even under full vision conditions can be attributed to the fact that participants were given movement time feedback after each trial and encouraged to try to improve their MT performance.

In order to obtain a more fine-grained index of aiming accuracy/consistency, we also analyzed variable error as a measure of effective target width (Schmidt et al., 1979). As well as a main effect for Vision Condition, $\mathrm{F}(2,55)=69.88, p<.001$, this analysis yielded a Group by Block by Vision condition interaction, $\mathrm{F}(8,220)=2.08, p<.05$. Overall, participants exhibited less variable error with V $(4.88 \mathrm{~mm})$ than with $\mathrm{NV}(6.29 \mathrm{~mm})$. As is evident in Figure 3, vision vs no vision differences were relatively consistent across practice for participants in the B and NK groups. For PK participants, large vision-no vision differences in Blocks 1 and 2 disappeared as practice progressed.

\section{Acquisition-Movement Trajectory}

The peak velocity analysis yielded only a Group by Block interaction, $\mathrm{F}(8,220)=2.04, p<.05$. Participants in the PK group increased their peak velocities with practice $(\mathrm{B} 1=2.61$ $\mathrm{m} / \mathrm{s}, \mathrm{B} 2=2.72 \mathrm{~m} / \mathrm{s}, \mathrm{B} 3=2.74 \mathrm{~m} / \mathrm{s}, \mathrm{B} 4=2.79 \mathrm{~m} / \mathrm{s}, \mathrm{B} 5=2.85$ $\mathrm{m} / \mathrm{s}$ ) while there was no change in the other two groups (NPK: $\mathrm{B} 1=2.38 \mathrm{~m} / \mathrm{s}, \mathrm{B} 2=2.35 \mathrm{~m} / \mathrm{s}, \mathrm{B} 3=2.36 \mathrm{~m} / \mathrm{s}, \mathrm{B} 4=2.36 \mathrm{~m} / \mathrm{s}$,

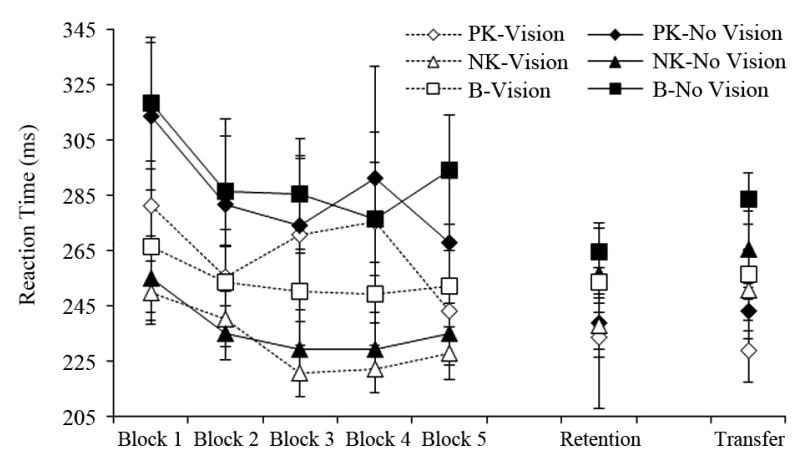

Figure 1.

Mean reaction time (ms) for the acquisition, retention and transfer blocks plotted as a function of prior knowledge group (PK, NK, B) and vision condition (vision, no vision). Error bars represent standard error of the mean.

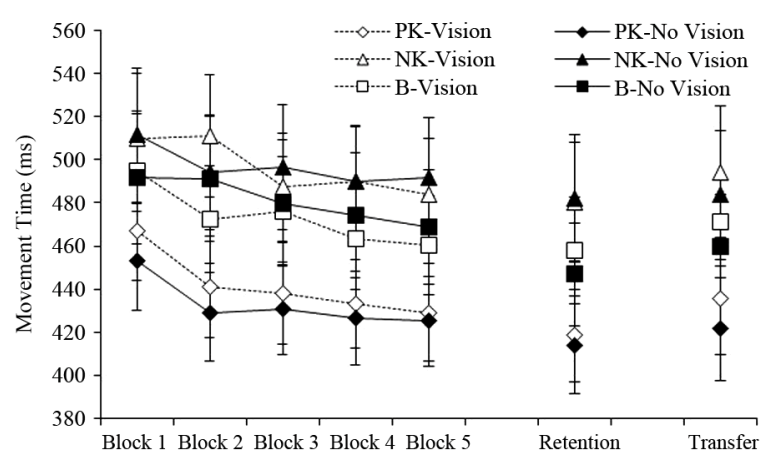

Figure 2.

Mean movement time (ms) for the acquisition, retention and transfer blocks plotted as a function of prior knowledge group (PK, NK, B) and vision condition (vision, no vision). Error bars represent standard error of the mean.

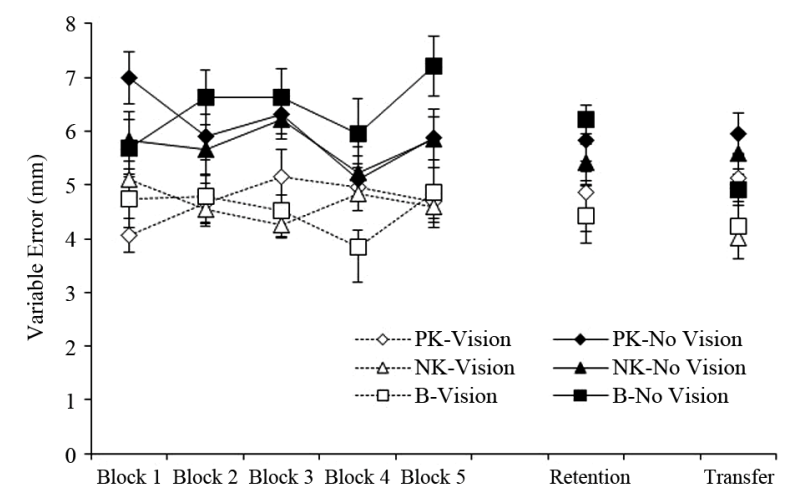

Figure 3.

Mean variable error $(\mathrm{mm})$ for the acquisition, retention and transfer blocks plotted as a function of prior knowledge group (PK, NK, B) and vision condition (vision, no vision). Error bars represent standard error of the mean.

$\mathrm{B} 5=2.37 \mathrm{~m} / \mathrm{s} ; \mathrm{B}: \mathrm{B} 1=2.54 \mathrm{~m} / \mathrm{s}, \mathrm{B} 2=2.52 \mathrm{~m} / \mathrm{s}, \mathrm{B} 3=2.56 \mathrm{~m} / \mathrm{s}$, $\mathrm{B} 4=2.45 \mathrm{~m} / \mathrm{s}, \mathrm{B} 5=2.55 \mathrm{~m} / \mathrm{s})$. The time to peak velocity analysis revealed main effect for Block, $\mathrm{F}(4,220)=7.63, p<.001$, and a Group by Block by Vision condition interaction, $\mathrm{F}(8,220)$ $=2.04, p<.05$ (see Table 1$)$. 
Table 1.

The mean ( \pm SE) time to peak velocity (ttPV), time after peak velocity (taPV), movement time (MT) and proportional time spent after peak velocity for aims performed with vision and without vision for each of the acquisition, retention and transfer blocks.

\begin{tabular}{|c|c|c|c|c|c|}
\hline & Block & $\mathrm{ttPV}(\mathrm{ms})$ & $\begin{array}{c}\text { taPV } \\
\text { (ms) }\end{array}$ & $\begin{array}{l}\text { MT } \\
\text { (ms) }\end{array}$ & $\begin{array}{c}\text { Proportional Time } \\
\text { Spent after } \\
\text { PV (\%) }\end{array}$ \\
\hline \multirow[t]{7}{*}{ Vision } & 1 & $215(7)$ & 278 (10) & 492 (15) & $56.5(.01)$ \\
\hline & 2 & $208(7)$ & 266 (9) & 475 (15) & $56.1(.01)$ \\
\hline & 3 & $211(8)$ & $256(8)$ & 467 (14) & $55.0(.01)$ \\
\hline & 4 & $208(8)$ & $254(7)$ & $462(14)$ & $55.0(.01)$ \\
\hline & 5 & $201(7)$ & 257 (8) & $458(14)$ & $56.2(.01)$ \\
\hline & Retention & $202(8)$ & $263(8)$ & $452(14)$ & $56.8(.01)$ \\
\hline & Transfer & $198(7)$ & $262(8)$ & 467 (15) & $56.8(.01)$ \\
\hline \multirow[t]{7}{*}{ No Vision } & 1 & $220(8)$ & 265 (10) & $486(16)$ & $54.4(.01)$ \\
\hline & 2 & $216(8)$ & 256 (9) & $472(15)$ & $54.3(.01)$ \\
\hline & 3 & $213(8)$ & $256(9)$ & $469(16)$ & $54.6(.01)$ \\
\hline & 4 & $209(8)$ & 254 (8) & $464(15)$ & $54.8(.01)$ \\
\hline & 5 & $202(7)$ & $260(9)$ & $462(15)$ & $56.4(.01)$ \\
\hline & Retention & $199(8)$ & $261(8)$ & 448 (15) & $56.7(.01)$ \\
\hline & Transfer & $200(7)$ & 257 (9) & 455 (15) & $57.1(.01)$ \\
\hline
\end{tabular}

Overall, participants systematically decreased the time taken to reach peak velocity as practice progressed. This decrease in time, along with the increase in the magnitude of peak velocity in the PK group explains the majority of the decrease in MT with practice. As is apparent in Figure 4, participants in the PK and NK groups showed similar improvements in the two vision conditions over practice. Participants in the Blocked group however improved most under no vision conditions with the only significant difference between the two vision conditions occurring in the first two blocks of practice.

Changes in the symmetry of the velocity profiles with practice were confirmed by analysis of the proportional time spent after peak velocity. Specifically a small but significant main effect for Block, $\mathrm{F}(4,220)=3.86, p<.01$, revealed that participants spent more proportional time after peak velocity in Block 5 than Blocks 1 - 4 (Tukey HSD, $p<.05$; see Figure 5). This analysis also yielded a main effect for Vision Condition, $F(1,55)$ $=8.05, p<.01$, and a Block by Vision Condition interaction, $\mathrm{F}(4,220)=3.44, p<.01$. Overall, participants spent more proportional time after peak velocity when vision was available but this difference was only significant for the first two blocks of trials. With practice the symmetry of the no vision velocity profiles became more similar to the profiles associated with full vision (Figure 5).

In order to understand how spatial variability unfolds over the course of the entire movement we also calculated the standard deviations of the distance covered in the primary direction of the movement at 4 important Kinematic Markers (e.g., peak acceleration, peak velocity, peak deceleration and movement termination; see Elliott \& Hansen, 2010; Khan et al., 2006). These within-participant standard deviations were submitted to a 3 Group by 4 Kinematic Marker by 5 Block by 2 Vision Con- dition mixed analysis of variance. This analysis revealed only a main effect for Kinematic Marker, $\mathrm{F}(3,165)=100.47, p<.001$, and a Kinematic Marker by Vision Condition interaction, $\mathrm{F}(3,165)=6.52, p<.001$. As is evident in Figure 6, participants exhibited similar degrees of spatial variability under vision and no vision conditions at peak acceleration and peak velocity. They were significantly more variable when vision was available at peak deceleration than in the no vision condition, however this situation reversed itself during the final

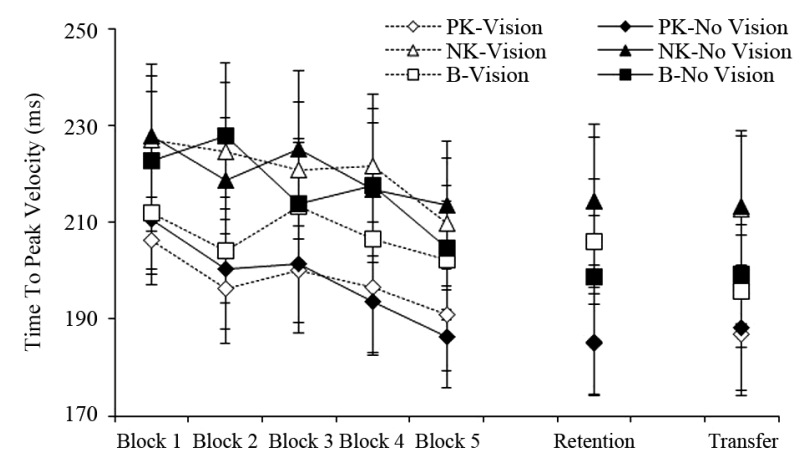

Figure 4.

Mean time to peak velocity (ms) for the acquisition, retention and transfer blocks plotted as a function of prior knowledge group (PK, NK, B) and vision condition (vision, no vision). Error bars represent standard error of the mean.

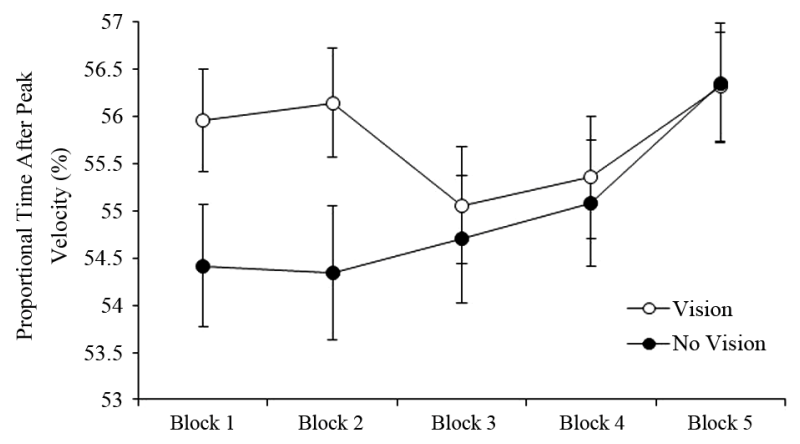

Figure 5.

Mean proportional time after peak velocity for acquisition plotted as a function of block and vision condition. Error bars represent standard error of the mean.

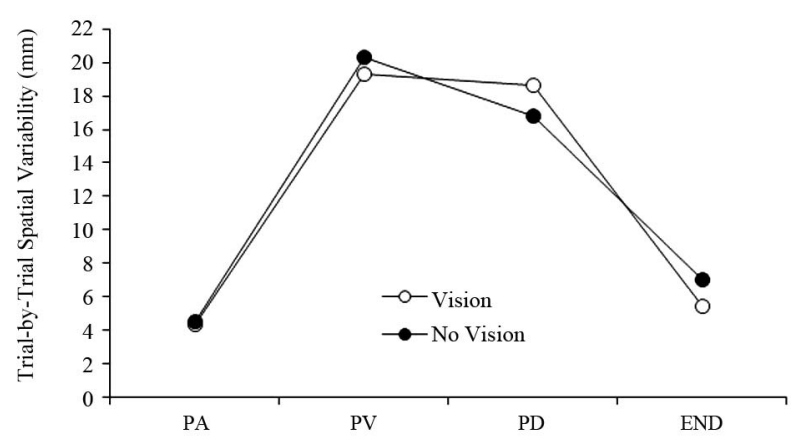

Figure 6.

Mean trial-by-trial spatial variability (mm) for acquisition plotted as a function of kinematic marker (PA, PV, PD, END) and vision condition (vision, no vision). 
phase of the movement. Presumably the greater variability associated with vision at peak deceleration reflect discrete corrective processes designed to bring the limb onto the target. These processes were effective as evidenced in the end point variability (see also VE analysis). Contrary to our expectations, Group had no impact on this spatial variability analysis. Thus participants were benefiting from late visual information regardless of their expectations.

\section{Retention/Transfer-Outcome Measures}

In order to examine not only retention and transfer per se, but also how performance changed between the performance conditions available during acquisition and the retention/transfer conditions, we decided to include the last block of acquisition in this set of analyses. Thus for the majority of dependent variables, we conducted a 3 Group (PK, NK, B) by 3 Phase (A5, R, $\mathrm{T}$ ) by 2 Vision Condition $(\mathrm{V}, \mathrm{N})$ mixed analysis of variance where A5 represents the last block of Acquisition, R represents the retention condition in which the target size remained the same and $\mathrm{T}$ the transfer condition in which the target size was smaller.

The reaction time analysis yielded a main effect for Vision Condition, $\mathrm{F}(1,55)=32.06, p<.001$ and a Group by Phase interaction, $\mathrm{F}(4,110)=3.88, p<.01$. Overall participants were faster initiating their movements when vision was available during movement execution (243 ms) than when it was eliminated (261 ms). This finding makes sense given that in all but the A5-NK situation, participants were aware that they would have vision available to regulate their aiming. The interaction is apparent in Figure 1. Participants in the Blocked group exhibited long, but consistent RTs across the 3 performance situations. The absence of any change reflects that fact that, for this group, there is no change in the visual or prior knowledge conditions between acquisition and retention/transfer. Participants in the PK group became significantly faster at initiating their movements during retention and transfer. Presumably this is because these participants are accustomed to using prior knowledge and in retention and transfer had the added advantage of maintaining their strategy from trial-to-trial. It was also the case that these participants had a variable practice structure. This trial-to-trial variability in the movement preparation demands during acquisition (i.e., preparing of vision and no vision trials differently) may have contributed to superior performance during retention (e.g., Lee \& Magill, 1983). NK participants became slower in retention and transfer. This increase in time for NK participants could partly reflect the new situation they were faced with. That is, unlike acquisition they were now able to plan their movements knowing what type of feedback they would receive, but had no prior practice in doing so. Thus they required time to prepare their movements. As well, their exposure to a constant practice structure during acquisition would also be expected to interfere with long-term retention and transfer.

The movement time analysis revealed a main effect for Phase, $\mathrm{F}(2,110)=3.93, p<.05$, as well as a Phase by Vision Condition interaction, $\mathrm{F}(2,110)=4.31, p<.05$. Overall, participants were faster during retention ( $450 \mathrm{~ms}$ ) than they were during A5 (460 ms) or $\mathrm{T}(461 \mathrm{~ms})$. The interaction reflects the fact that when aiming at the smaller target during transfer, participants took more time when they had vision available $(467 \mathrm{~ms})$ than when they knew it would be eliminated ( $455 \mathrm{~ms}$; A5-V = 458 ms, A5-NV = $462 \mathrm{~ms}, \mathrm{R}-\mathrm{V}=452 \mathrm{~ms}$, R-NV = $448 \mathrm{~ms}$; Figure 2). It appears that regardless of the type of training participants received they adjusted their movement times to reflect the new visual and target circumstance they are faced with.

The variable error analysis revealed main effects for Vision Condition, $\mathrm{F}(1,55)=38.55, p<.001$, and Phase, $\mathrm{F}(2,110)=$ $7.60, p<.001$, as well as a Group by Phase interaction, $\mathrm{F}(4,110)$ $=3.46, p=.01$. Overall, participants were more consistent with vision $(4.79 \mathrm{~mm})$ than without vision $(5.92 \mathrm{~mm})$. The Group by Phase interaction is evident in Figure 3. As one might expect, the PK group maintained their solid performance in acquisition through both retention and transfer. The NK group improved slightly from A5 to retention and transfer. This change could reflect the improved performance circumstance for these participants who now had prior information about the feedback available. The most robust result was the large improvement shown by the participants who received Blocked training. Of course they were the only participants to perform under exactly the same conditions available during acquisition. The improvement in end point consistency could reflect the fact that during retention and transfer participants are no longer given movement time feedback (cf. Block 5 of acquisition). Perhaps, this situation allows them to focus their attention on movement accuracy.

The error analysis yielded only main effects for Phase, $\mathrm{F}(2,110)=65.71, p<.001$, and Vision Condition, $\mathrm{F}(1,55)=$ $17.40, p<.001$. As one would expect, participants had more trouble hitting the smaller target during transfer (48.0\% misses) than they did the larger target (A5 $=21.4 \%, \mathrm{R}=23.7 \%$ ). Of course they also exhibited less error when vision was available (27.2\% misses) than when it was eliminated upon movement initiation (34.9\% misses).

\section{Retention/Transfer-Movement Trajectory}

The peak velocity analysis yielded only a main effect for Phase, $\mathrm{F}(2,110)=6.95, p<.01$. Participants achieved higher peak velocities during A5 $(2.84 \mathrm{~m} / \mathrm{s})$ than during retention $(2.42$ $\mathrm{m} / \mathrm{s}$ ). The PVs during transfer were intermediate and not significantly different from the other two values $(2.54 \mathrm{~m} / \mathrm{s})$. The time to peak velocity analysis failed to reveal any significant effects (grand mean $=200 \mathrm{~ms}$ ). The proportional time after peak velocity analysis also failed to reveal any significant effects. Under all conditions participants spent slightly more time after PV than before PV (grand mean = .567). It would appear that the movement time effects reported earlier result from the combined influence of the time before and after peak velocity. As well, both practice and the similarity of performance conditions reduced any temporal effects associated vision condition or group effects that were evident during acquisition.

Once again we hoped to gain some insight into the spatial characteristics of the limb trajectories by examining the standard deviations of spatial position in the primary direction of the movement at PA, PV, PD and the end of the movement. Thus we conducted a 3 group by 4 Kinematic Marker by 3 Phase by 2 Vision Condition mixed analysis of variance on these standard deviations. This analysis revealed a main effect for Kinematic Marker, $\mathrm{F}(3,165)=93.42, p<.001$, as well as interactions involving Kinematic Marker and Vision Condition, $\mathrm{F}(3,165)=6.66, p<.001$, and Phase, Kinematic Marker and Vision Condition, $\mathrm{F}(6,330)=3.46, p<.01$. As is evident in Figure 7, during the last block of acquisition there was very 

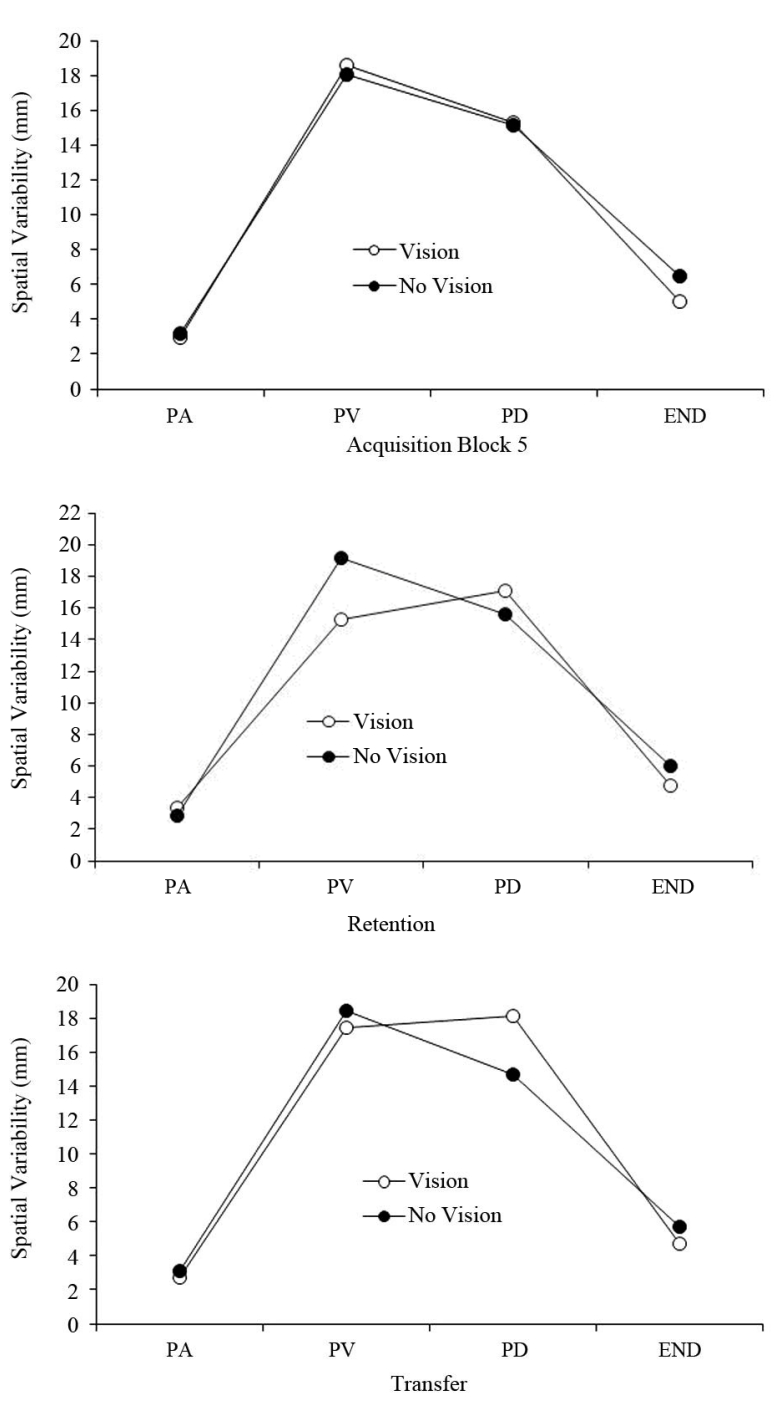

Figure 7.

Mean trial-by-trial spatial variability (mm) for the last acquisition block, retention block and transfer block plotted as a function of vision condition (vision, no vision).

little difference in variability between vision and no vision over the course of the trajectory except for at the movement END point. Thus visual regulation appears to have occurred primarily after peak deceleration. For retention and transfer, where all participants are performing with prior knowledge about feedback, variability is higher under no vision conditions at peak velocity than when vision is available. However, in both retention and transfer there is a significant reduction in variability under no vision conditions between peak velocity and peak deceleration. When vision is available higher standard deviations at peak deceleration could reflect corrective processes that are effective in providing a visual advantage by the END of the movement. Once again participants in all 3 groups appear to be taking advantage of the visual information and advance information available to them during retention and transfer rather than being disadvantaged by the information associated with their particular acquisition group.

Interestingly although training group did have an impact on some of the outcome findings during retention and transfer (i.e., $\mathrm{RT}$ and VE), there is very little indication from the kinematic data that participants are executing their movements differently depending on the type of training they received. This suggests that participants were extremely flexible in their strategic approach to the task and were able to alter their planning and execution procedures in order to meet the new visual and prior knowledge circumstance.

\section{Discussion}

The purpose of this study was to determine if prior knowledge about the availability of visual information for limb control impacts the manner in which adult performers learn to optimize rapid goal-directed aiming movements. Although participants in all 3 groups decreased both their vision and no vision movement times over the 5 acquisition blocks, this improvement was achieved in slightly different ways. Consistent with previous research (Hansen et al., 2006; Khan et al., 2002), prior knowledge about vision did have an influence on both the time taken for movement planning and the characteristics of the movement trajectory during skill acquisition. Specifically, performers who were aware of the visual condition prior to a trial (e.g., PK and B participants) took less time to prepare their movements with vision than no vision. Presumably they took less time for planning because they knew they would have vision available for online regulation during movement execution, and thus an opportunity to correct any errors in the movement trajectory. Participants in the no prior knowledge group took a similar amount of time for both vision and no vision planning, but unexpectedly were quicker than participants in the other two groups. This overall advantage could reflect the fact that these participants had no reason to change their movement preparation strategy either from trial-to-trial (cf. PK group) or block-to-block (cf. B group).

During acquisition, prior knowledge also had an impact on the characteristics of the movement trajectory. Specifically participants in the prior knowledge group decreased their movement times by increasing limb velocities while, for the other two groups, improvement was related to the time taken to achieve peak velocity. Participants in the Blocked group were particularly adept at improving their no vision performance early in acquisition. Thus, at least for no vision situations, there does appear to be an advantage associated with performing the same type of trial several times in a row. Specifically, these participants were able to use feedback from trial $\mathrm{N}$ to improve their performance in trial $\mathrm{N}+1$ (Cheng et al., 2008; Elliott et al., 2004; Mon-Williams \& Bingham, 2007).

Although prior knowledge did have an impact on both movement planning and the characteristics of the limb trajectory, it is important to note that there were also systematic advantages associated with having vision available regardless of prior knowledge condition. Specifically, participants hit the target more often and exhibited less variable error when vision was available than when it was eliminated upon movement initiation. Moreover, the spatial variability analysis of the acquisition data yielded some interesting results. Regardless of prior knowledge group, participants exhibited greater spatial variability at peak deceleration and less spatial variability at the end of the movement (i.e., variable error) when vision was available than when it was eliminated upon movement initiation. In the past, we have speculated that some of the greater vari- 
ability at peak deceleration associated with vision could be due to less precise planning. Performers take this strategic approach because vision will be available for late online corrective processes to operate (Elliott et al., 2010; Khan et al., 2002). Here however, participants in the no prior knowledge group did not know in advance whether or not vision would be available. Thus, the greater variability at peak deceleration associated with vision probably reflects trial-to-trial differences between vision and no vision in corrective procedures designed to bring the limb onto the target at movement termination. These differences in spatial variability at peak deceleration indicate that, regardless of prior knowledge, visual corrective processes begin to operate sometime before peak deceleration. As we have proposed elsewhere (Elliott et al., 2010; Grierson \& Elliott, 2009), this type of evidence for early online control indicates that the primary submovement (Meyer, Abrams, Kornblum, Wright, \& Smith, 1988; Woodworth, 1899) might not be as ballistic as previously thought. It would seem that regardless of the movement plan, participants recognize the advantages associated with the utilization of visual feedback when it becomes available (even unexpectedly) and adjust their control processes accordingly. The variability data suggest that these corrective processes begin to operate sometime between peak velocity and peak deceleration.

The vision-no vision performance effects associated with acquisition were also apparent in retention and transfer. Specifically, with prior knowledge available to all participants, movement planning continued to take more time under no vision than vision conditions. Once again we speculate that more precise planning is undertaken when participants are aware that they will not have the opportunity for visual online control. This extra planning takes time. As well, participants from all groups terminated their movements more consistently and hit the target more often when vision was available than when it was eliminated upon movement initiation. Moreover, there was an additional performance effect associated with our visual manipulation when a smaller target was introduced in our transfer situation. In this context, participants exhibited longer movement times under vision than no vision conditions. Presumably this extra time reflects the added corrective procedures required to hit the smaller target (see also variable error and error analysis).

It should be pointed out that when vision was eliminated upon movement initiation both visual information about the limb and the target was removed. This means that participants were more dependent on proprioceptive information and feedforward information about limb position for online control but were also dependent on a visual representation of the target position (Ghahramani, Wolpert, \& Jordan, 1996). Although memory for target position appears to be robust over short novision delays (Carlton, 1981; Elliott \& Madalena, 1987), in future research it would be appropriate to examine limb and target effects separately (Hayes, Elliott, \& Bennett, 2010).

During retention and transfer, training group did have an impact on reaction time. Specifically, participants in the PK group exhibited a significant decrease in RT when they were given the added advantage of blocked vision-no vision protocols. Participants in the NK group exhibited an increase in RT during retention and transfer. Presumably this is because they now had additional information for movement preparation (i.e., prior knowledge about feedback), but no experience using that information.
The most interesting group findings during retention and transfer were related to variable error. Although the prior knowledge group maintained their variable error performance during retention and transfer, the no prior knowledge group improved slightly under both vision and no vision conditions. Presumably this change was due to the improved performance situation. That is, these participants now had prior knowledge about the availability of vision and were also able to use information from trial $\mathrm{N}$ to plan trial $\mathrm{N}+1$. Unexpectedly the participants in the Blocked group exhibited the most improvement during retention and transfer even though they were performing under the same conditions as acquisition. Although initially surprising, this finding makes sense if one considers that movement time feedback was also eliminated during retention and transfer. Because participants in the Blocked group were performing under exactly the same feedback and prior knowledge conditions as acquisition, they would be expected to have no trouble maintaining their end point error performance. The added advantage may be associated with the elimination of movement time feedback, allowing these participants, and all other participants, to concentrate more on movement accuracy. Unlike the PK and NK participants, performers in the Blocked group also had the experience from acquisition of making use of trial $\mathrm{N}$ information about accuracy to improve trial $\mathrm{N}+1$ performance.

Although acquisition group did have an impact on both RT and $\mathrm{VE}$, the movement kinematics during retention and transfer were surprisingly similar for all groups of participants. It would appear that performers, from all 3 groups, were able to quickly adjust the characteristics of their movement trajectories to meet the current prior knowledge and visual circumstance. With respect to vision condition, we once again found evidence for early online control when vision was available. In fact during retention and transfer, spatial variability differences between vision and no vision appeared as early as peak velocity, indicating that sometime before peak velocity participants were using visual feedback to adjust their movement trajectories. This influence of vision was earlier than what was seen in acquisition, and perhaps reflects the fact that participants in all groups now had prior knowledge about the availability of visual and/or just proprioceptive feedback.

Although prior knowledge has a robust impact on performance (Elliott \& Allard, 1985; Hansen et al., 2006; Khan et al., 2002; Zelaznik et al., 1983), it has a more subtle effect on motor learning. Specifically, adult performers appear to be adept at quickly adjusting the characteristics of their aiming movements to make maximal use of vision and the other information available to them (Elliott et al., 1995; Elliott et al., 2004). If, as we have suggested elsewhere (Elliott et al., 2010), rapid visual regulation depends on the development of viable internal models of limb control that include expected sensory consequences, then having experience with visual feedback, regardless of prior expectancy, may be enough. Perhaps this is not surprising given that recent work on action-observation indicates that general procedures for visual feedback regulation do not even depend on physical performance. Specifically, a performer can develop at least some of these representations via observation (Hayes et al., 2010).

What is clear from this work is that regardless of expectancy, vision is a powerful mediator of rapid goal-directed movement. In this experiment, we have evidence to indicate that, when feedback is expected (i.e., in this study during retention and 
transfer), visual regulation begins to occur prior to peak velocity. Thus this regulation occurs during the accelerative phase of the primary submovement. Consistent with our recent multiple process model of goal-directed aiming (Elliott et al., 2010), there appears to be more than one type of visual online control. The type of early control evident here probably involves a comparison of dynamic visual feedback with feedback expectancies (cf. Meyer et al., 1988; Woodworth, 1899). Late online control occurs after the primary submovement is complete. It involves a comparison of limb and target positions. It is this type of late regulation that contributes to vision vs. no vision differences when there is uncertainty about the availability of visual feedback. That is, participants prepare for a no vision scenario, as evidenced by RT, but then still benefit from vision late in the movement when it is available, as evidenced by the spatial variability and variable error results.

In summary, expectancy about the availability of visual feedback impacts motor performance (see also Elliott \& Allard, 1985; Hansen et al., 2006; Zelaznik et al., 1983). The impact of expectancy on motor learning however is more complex. Our findings suggest that performers are extremely flexible in their ability to use vision and other sensory information to optimize performance. Specifically, adult performers appear able to change their strategic behavior quickly to accommodate new sensory and prior knowledge conditions.

\section{Acknowledgements}

This research was supported by the Natural Sciences and Engineering Research Council of Canada.

\section{REFERENCES}

Bard, C., Hay, L., \& Fleury, M. (1985). Role of peripheral vision in the directional control of rapid aiming movements. Canadian Journal of Psychology, 39, 151-161. doi:10.1037/h0080120

Carlton, L. G. (1981). Visual information: The control of aiming movements. Quarterly Journal of Experimental Psychology, 33A, 87-93.

Cheng, D. T., Luis, M., \& Tremblay, L. (2008). Randomizing visual feedback in manual aiming: Reminiscence of the previous trial condition and prior knowledge of feedback availability. Experimental Brain Research, 189, 403-410. doi:10.1007/s00221-008-1436-3

Elliott, D., \& Allard, F. (1985). The utilization of visual feedback information during rapid pointing movements. Quarterly Journal of Experimental Psychology, 37(A), 407-425.

Elliott, D., Chua, R., Pollock, B. J., \& Lyons, J. (1995). Optimizing the use of vision in manual aiming: The role of practice. Quarterly Journal of Experimental Psychology: Human Experimental Psychology, 48(A), 72-83. doi:10.1080/14640749508401376

Elliott, D., \& Hansen, S. (2010). Visual regulation of aiming: A comparison of methods. Behavior Research Methods, 42, 1087-1095. doi:10.3758/BRM.42.4.1087

Elliott, D., Hansen, S., Grierson, L. E. M., Lyons, J., Bennett, S. J., \& Hayes, S. J. (2010). Goal-directed aiming: Two components but multiple processes. Psychological Bulletin, 136, 1023-1044. doi:10.1037/a0020958

Elliott, D., Hansen, S., Mendoza, J., \& Tremblay, L. (2004). Learning to optimize speed, accuracy, and energy expenditure: A framework for understanding speed-accuracy relations in goal-directed aiming. Journal of Motor Behavior, 36, 339-351.
doi:10.3200/JMBR.36.3.339-351

Elliott, D., Helsen, W. F., \& Chua, R. (2001). A century later: Woodworth's two-component model of goal directed aiming. Psychological Bulletin, 127, 342-357. doi:10.1037/0033-2909.127.3.342

Elliott, D., \& Madalena, J. (1987). The influence of premovement visual information on manual aiming. Quarterly Journal of Experimental Psychology: Human Experimental Psychology, 39A, 541-559. doi:10.1080/14640748708401802

Ghahramani, Z., Wolpert, D. M., \& Jordan, M. I. (1996). Generalization of local remappings of the visuomotor coordinate transformation. Journal of Neuroscience, 16, 7085-7096.

Grierson, L. E. M., \& Elliott, D. (2009). Goal-directed aiming and the relative contribution of two online control processes. American Journal of Psychology, 122, 309-324.

Hansen, S., Glazebrook, C., Anson, J. G., Weeks, D. J., \& Elliott, D. (2006). The influence of advance information about target location and visual feedback on movement planning and execution. Canadian Journal of Experimental Psychology, 60, 200-208. doi:10.1037/cjep2006019

Hayes, S. J., Elliott, D., \& Bennett, S. J. (2010). General motor representations are developed during action-observation. Experimental Brain Research, 204, 199-206. doi:10.1007/s00221-010-2303-6

Henriques, D. Y., Klier, E. M., Smith, M. A., Lowy, D., \& Crawford, J. D. (1998). Gaze-centred remapping of remembered visual space in an open-loop pointing task. Journal of Neuroscience, 18, 1583-1594.

Kawato, M., \& Wolpert, D. M. (1998). Internal models for motor control. Novartis Foundation Symposium, 218, 219-304.

Keele, S. W., \& Posner, M. I. (1968). Processing visual feedback in rapid movements. Journal of Experimental Psychology, 77, 155-158. doi: $10.1037 / \mathrm{h} 0025754$

Khan, M. A., Elliott, D., Coull, J., Chua, R., \& Lyons, J. (2002). Optimal control strategies under different feedback schedules: Kinematic evidence. Journal of Motor Behavior, 34, 45-57. doi:10.1080/00222890209601930

Khan, M., Franks, I. M., Elliott, D., Lawrence, G. P., Chua, R., Bernier, P. M., Hansen, S., \& Weeks, D. J. (2006). Inferring online and offline processing of visual feedback in target-directed movements from kinematic data. Neuroscience and Biobehavioral Reviews, 30, 11061221. doi:10.1016/j.neubiorev.2006.05.002

Lee, T. D., \& Magill, R. A. (1983). The locus of contextual interference in motor skill acquisition. Journal of Experimental Psychology: Learning, Memory and Cognition, 9, 730-746. doi:10.1037/0278-7393.9.4.730

Meyer, D. E., Abrams, R. A., Kornblum, S., Wright, C. E., \& Smith, J. E. K. (1988). Optimality in human motor performance: Ideal control of rapid aimed movements. Psychological Review, 95, 340-370. doi:10.1037/0033-295X.95.3.340

Milgram, P. (1987). A spectacle-mounted liquid-crystal tachistoscope. Behavior Research Methods, Instruments and Computers, 19, 449456. doi:10.3758/BF03205613

Mon-Williams, M., \& Bingham, G. P. (2007). Calibrating reach to visual targets. Journal of Experimental Psychology: Human Perception and Performance, 33, 645-656. doi:10.1037/0096-1523.33.3.645

Schmidt, R. A., Zelaznik, H. N., Hawkins, B., Frank, J. S., \& Quinn, J. T. (1979). Motor output variability: A theory for the accuracy of rapid motor acts. Psychological Review, 86, 415-451. doi:10.1037/0033-295X.86.5.415

Wolpert, D. M., \& Flanagan, J. R. (2001). Motor prediction. Current Biology, 11, R729-R732. doi:10.1016/S0960-9822(01)00432-8

Woodworth, R. S. (1899). The accuracy of voluntary movement. Psychological Review, 3, 1-119.

Zelaznik, H. N., Hawkins, B., \& Kisselburgh, L. (1983). Rapid visual feedback processing in single-aiming movements. Journal of Motor Behavior, 15, 217-236. 